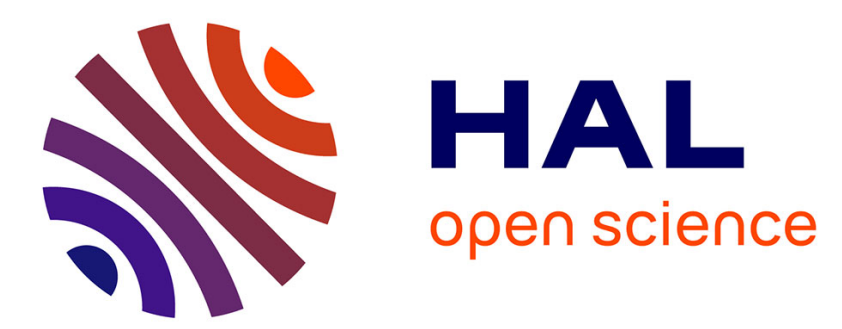

\title{
Interact With Me: An Exploratory Study on Interaction Factors for Active Physical Human-Robot Interaction
}

Yue Hu, Mehdi Benallegue, Gentiane Venture, Eiichi Yoshida

\section{To cite this version:}

Yue Hu, Mehdi Benallegue, Gentiane Venture, Eiichi Yoshida. Interact With Me: An Exploratory Study on Interaction Factors for Active Physical Human-Robot Interaction. IEEE Robotics and Automation Letters, 2020, 5 (4), pp.6764-6771. 10.1109/LRA.2020.3017475 . hal-02957318

\section{HAL Id: hal-02957318 https://hal.science/hal-02957318}

Submitted on 5 Oct 2020

HAL is a multi-disciplinary open access archive for the deposit and dissemination of scientific research documents, whether they are published or not. The documents may come from teaching and research institutions in France or abroad, or from public or private research centers.
L'archive ouverte pluridisciplinaire $\mathbf{H A L}$, est destinée au dépôt et à la diffusion de documents scientifiques de niveau recherche, publiés ou non, émanant des établissements d'enseignement et de recherche français ou étrangers, des laboratoires publics ou privés. 


\title{
Interact with me: an Exploratory Study on Interaction Factors for Active Physical Human-Robot Interaction
}

\author{
Yue $\mathrm{Hu}^{1, *}$, Mehdi Benallegue ${ }^{1}$, Gentiane Venture ${ }^{1,2}$, Eiichi Yoshida ${ }^{1}$
}

\begin{abstract}
In future robotic applications in environments such as nursing houses, construction sites, private homes, etc, robots might need to take unpredicted physical actions according to the state of the users to overcome possible human errors. Referring to these actions as active physical humanrobot interactions (active pHRI), in this paper, the goal is to verify the possibility of identifying measurable interaction factors that could be used in future active pHRI controllers, by exploring and analyzing the state of the users during active pHRI. We hypothesize that active physical robot actions can cause measurable alterations in the physical and physiological data of the users, and that these measurements could be interpreted with users' personality and perceptions. We design an experiment where the participant uses the robot to play a visual puzzle game, during which, the robot takes unanticipated physical actions. We collect physiological and physical data, as well as outcomes of two state-of-the-art questionnaires on the perceptions of robots, $\mathrm{CH}-33$ and Godspeed Series Questionnaires (GSQ), and a pre-experiment personality questionnaire, to relate the collected data with the users' perceptions and personality. The experiment outcomes show that we can extract a few factors related to personality, perception, physiological, and physical measurements. Even though we could not draw very clear correlations, these outcomes give fundamental insights for the design of novel pHRI experiments.
\end{abstract}

\section{INTRODUCTION}

Physical contact is an inevitable feature of many future robotics applications, where often, the interaction actions cannot be planned beforehand due to unpredicted events. Examples of environments and situations in which this may occur daily are nursing houses, construction sites, packaging lines, cooking help, and personal care.

When using robots in direct physical contact with humans, safety is one of the most important concerns. In 2016, the safety standards for collaborative robots ISO/TS 15066 [1] was introduced to ensure the physical safety of the users [2], [3]. However, physical safety should not be the only concern in human-robot interaction (HRI). As well known, humans give importance to emotions and perceptions, which are fundamental in human-human interactions, therefore perceptions and mental safety should also be considered in HRI [4].

\footnotetext{
1 CNRS-AIST JRL (Joint Robotics Laboratory), ence and Technology (AIST), Tsukuba, $\begin{gathered}\text { Industrial } \\ \text { Sci- }\end{gathered}$ yuehudieee.org, mehdi.benalleguedaist.go.jp, e.yoshidalaist.go.jp

2 Department of Mechanical Systems Engineering, Tokyo University of Agriculture and Technology, Tokyo, Japan venturedcc.tuat.ac.jp

* International Research Fellow of Japan Society for the Promotion of Science (JSPS, Postdoctoral Fellowships for Research in Japan (Standard)). This research was partly supported by Grant-in-Aid for JSPS Research Fellow (No. 18F18754) and JSPS Grant-in-Aid for Research (No. 17H01804)
}

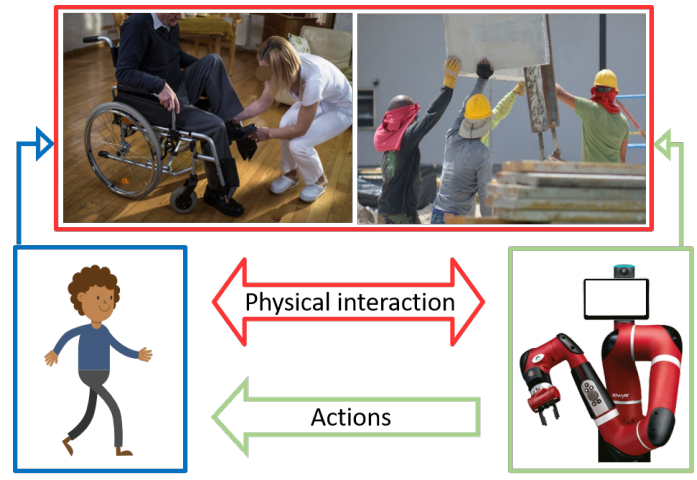

Fig. 1: Two possible scenarios for active pHRI: a robot helping in a nursing house and in a construction environment. In both cases, possible unpredictable events may occur and autonomous physical actions might be necessary.

In examples shown in Fig. 1, a collaborative manipulator could be used in nursing houses to help people in daily tasks such as wearing shoes, where the user could be uncooperative or need special care due to, e.g. mood swings, distraction, loss of stability, etc. In a construction site, robots could help the workers lifting and positioning pieces, where unpredicted events caused by wrong evaluations may occur. In both environments, people may be unaware of surrounding risks, make wrong decisions, and feel uncomfortable for being handled in certain ways. In our opinion, the robot should take actions that are corrective, taking into account the physical and mental states of the person, while still achieving the original task. As these could be unpredicted physical actions, the robot should also take into account the state of the users by "observing" their reactions to such probing actions.

The current safety standard imposes strict constraints on the performance of robots, inducing the robots to become rather "passive" and "slow" during interactions, moreover, it does not take into account users' mental state. We believe that new factors should be taken into account to boost the efficiency of human-robot interactions from both the robot and the human side. This means that the robot should become more active, i.e. with a controller that allows active interactions not only depending on users' physical state, but also perceptions and mental state.

In the context of HRI, robot controllers and human perceptions have been mostly treated in two main research directions, i.e. physical (pHRI) and social (sHRI), which have been evolving mostly in parallel without much intersections. In order to achieve our goals, we think it is necessary to combine tools from both sides.

In pHRI, it is implied that direct or indirect contacts 
between human users and robots may occur. Current stateof-the-art pHRI features topics such as co-manipulation [5], [6], collaborative object carrying [7], [8], the development of controllers with human-in-the-loop [9], [10], [11]. In most of these works, the main focus is given to the robot, i.e. the development of control frameworks in which the human is treated either as an external noise, an external force, or another "robot". Or, in the case of therapy and rehabilitation robotics [12], [13], focused on specific rehabilitation goals for the patient.

In contrast, sHRI focuses mainly on the human counterparts, trying to analyze their mental state, comfort, sense of safety, sociability, and in general users' perceptions. These are often studied via specially designed experiments involving little or no physical interactions with the robot, and a popular evaluation method is to use post-experiment questionnaires [14], [15], [16], [17]. Physiological measurements also proved to give useful insights, e.g. galvanic skin response (GSR) and electrocardiography [18], [19], [20] have been investigated to evaluate factors such as stress and anxiety, and eye gazing has been analyzed for engagement and proactiveness [21], [22]. Similar signals have also been used in Affective Computing to understand human emotions [23] and endowing machines with emotional intelligence.

A few works involved both physical and social interactions: evaluation of touch in a nursing situation [24], responses to hugging [25], rhythmic activities for teamwork [26]. The main focus of these works remains the evaluation of social perceptions, where the robot is designed as "social". In our case, we are interested in using tools from sHRI to evaluate the social aspects of people using also robots which have not been specially designed as "social", e.g. industrial manipulators, where the robot does not have to necessarily display social behaviors or emotional intelligence.

\section{A. Hypotheses and contributions}

We define active physical human-robot interaction (active pHRI) as an interaction during which robots may take physical actions on the human without prior notifications. Until now, in HRI there has been generally little or no expectation that the robot may take unpredicted physical actions, so the first step is to address the impact of such active actions on the user. For the robot to take the proper actions, the physical and mental state of the user needs to be measurable, i.e. a set of identifiable and quantitative interaction factors. For example, in a nursing house, if we were able to quantify and measure in real-time the anxiety of the user related to their perception of the robot actions, the robot could tune the motions to achieve different types of contact (softer, closer, stronger, etc), while maintaining the objective of achieving a specific task (e.g. helping to stand up).

The goal of this paper is to identify a first possible set of interaction factors by means of an active pHRI experiment. Our target is not the assessment of single perceptions (e.g. fear, satisfaction) or specific objectives (e.g. workload assessment, energy consumption). Rather, inspired by behavioral psychology, we hypothesize that it is possible to break down a more comprehensive human state into measurable factors, and that we can find relationships between the physical and physiological data and the perceptions and possibly the personality [27]. We formulate the following hypotheses:

H1 Unpredicted robot actions cause measurable alterations in the users' physical and physiological data;

H2 Measurable physical and physiological data could be explained with users' personality and perceptions of the robot.

To easily isolate possible factors, we designed an active pHRI experiment in which the user uses the robot to play a visual puzzle game. During the game, the robot takes active physical actions on the user similar to simple disturbances, of which the user is not informed beforehand, such that a natural reaction can be assessed. For the analysis, we collect physical and physiological data, personality, and post-experiment questionnaire outcomes related to the users' perceptions.

To avoid cultural dependencies, the experiments have been carried out only with Japanese nationals born and raised in Japan. We collected data from 23 participants (among which 17 were retained in this paper), then, we extracted a series of factors by post-processing the data. The major contributions and outcomes of this paper are:

- To the best of our knowledge, the first experiment addressing the analysis of the human state during active pHRI;

- First insights on measurable factors, despite the outcomes showing just a few relevant correlations between personality and user perceptions with physical and physiological data;

- Important insights for active pHRI scenarios and experiments analysis and design.

\section{B. Paper organization}

This paper is organized as follows: in section II we describe the details of the experiment, including the design choices, the control of the robot, the description of the setup, and the experiment protocol; in section III we illustrate the performed experiments, the factor extraction, and relevant factors; in section $[\mathrm{IV}$ we analyze and discuss the obtained results; in section $\mathrm{V}$ we summarize the outcomes and give an outlook of our next steps.

\section{EXPERIMENT DESCRIPTION}

The experiment has been designed to allow the users to have an intuitive direct physical interaction with the robot, and at the same time, allowing them to feel the action of the robot on the users. In the following subsections, we will illustrate the design choices, the control of the robot, the hardware equipment, and the experimental protocol.

\section{A. Design and tools choices}

Active pHRI scenarios could involve a high variety of factors, so we designed the first experiment to be a simple one, to allow for a straightforward identification of possible 


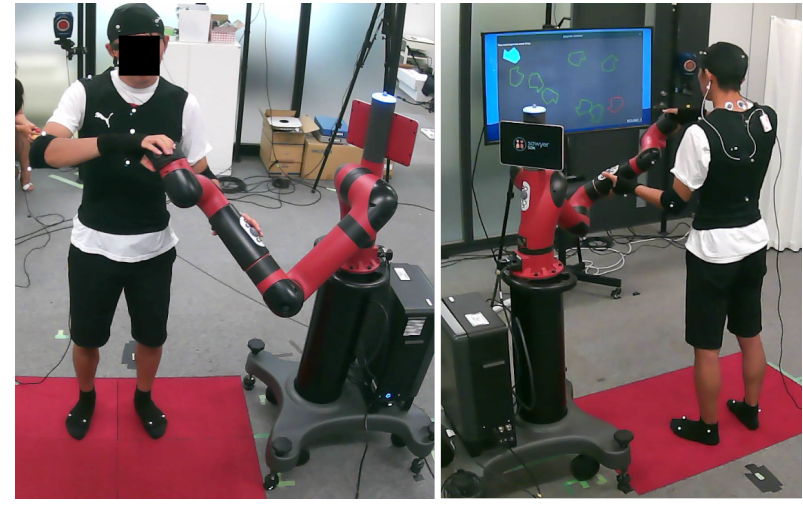

Fig. 2: The user uses the robot as a joystick to play the visual puzzle game.

factors. As shown in Fig. 2, during the experiment, the user has to use the robot as a joystick to play a visual puzzle game, shown on a screen. The user has to match the pieces by moving the end-effector (EE) of the robot in space, coming into an intuitive direct physical contact with the robot. During the interaction, the robot performs actions on the user by pushing or pulling with an active force. The game and the action can be seen in the video attachment. The visual game approach is similar to rehabilitation robots [12], where patients often play visual games using a robot arm with direct physical contact. However, in these cases, the interaction is guided and the objective is to perform specific rehabilitation programs via the game, with little focus on social perceptions and mental state.

As stated in the hypotheses, we aim at measuring the physical and physiological data of the users. Among the available physiological measurements, we decided to adopt GSR, Photoplethysmography (PPG), and eye-tracking. GSR is a well-known indicator of arousal, its variations allow us to measure the intensity of arousal to stimuli [28], [29]. The PPG measurement is used as an alternative to the Heart Rate Variation (HRV), which is another popular indicator. From the PPG signal, it is possible to extract the Pulse Rate Variability (PRV) [30], which is related to mental stress, where higher PRV (with respect to the baseline conditions) indicates higher mental stress [31], [32]. Eye blinking frequency (EBR) and eye blinking duration (EBD) have been related to mental load, where higher EBR and shorter EBD correspond to higher mental load [33], [34]. From a standard eye tracker, it is also possible to obtain the gazing direction, which is another important indicator [21], [22].

For the physical data, we collect motion data, ground reaction forces, and interaction forces with the robot. From these data, it is possible to extract a series of information regarding the overall behavior of the user towards the robot (e.g. distances, forces exerted).

One of the most common tools used in sHRI to evaluate the post-experiment perceptions of the users towards the robot is questionnaire. Among the questionnaires available in the literature, we decided to adopt $\mathrm{CH}-33$, which has been established as a measurement of psychological safety towards robots [4], and the Godspeed Series Questionnaire (GSQ) [35], which is one of the most popular questionnaires in HRI [36]. As personality has been shown to be relevant in HRI [27], [37], we decided to adopt a simplified version of the Big Five, one of the most commonly used personality traits questionnaire [38]. All the questionnaires are available online at [39].

\section{B. Robot control}

To play the game using the robot, the user has to come into contact with its EE. The users are free to move the robot in any direction, and the 3D Cartesian space motion of the robot $\mathrm{EE}$ is projected into the $2 \mathrm{D}$ movement of the target piece. The rotation of the piece is a direct mapping of the joint angle of the last joint before the EE. If the robot is released, it stops all the motions.

We assume the robot to be torque-controlled when the user is in contact, and in position control otherwise. We implement a controller that runs at a frequency of 100 $\mathrm{Hz}$, implemented using a Quadratic-Programming (QP) [40] formulation which allows us to take into account joint limits, joint velocity limits, and joint torque limits. During the game, the robot takes a timed action on the user, i.e. every $\Delta T$, an active force $\mathbf{f}_{a}$ is applied. The direction of $\mathbf{f}_{a}$ is opposite to the estimated EE velocity direction at the instant the active force starts to be applied. The magnitude is proportional to the force exerted by the user on the EE, bounded in specified minimum and maximum, to ensure that the force is perceived yet at the same time safe for the user.

The active force is applied only when the user is interacting, therefore when the robot is in torque control. The time interval $\Delta T$ is counted only over the time during which the cuff is pressed, i.e. for the user, the interval between each active force is unknown. The active force is not applied instantaneously, but over a duration of $\Delta T_{a} \ll \Delta T$.

\section{Equipment and sensors}

The robot we use for the experiment is a Sawyer collaborative robot (Rethink Robotics), which has 7 degrees of freedom. It is torque-controlled and equipped with Series Elastic Actuators, and it complies with the safety standards ISO/TS 15066. The robot can avoid self-collisions, it has joint torque sensors in all the joints, which are also used to estimate the external wrench at the EE. With Sawyer, to trigger the torque control mode, the user has to press the cuff button located at the EE, as shown in Fig. 2 .

To measure the user's state during the experiment, we use the following equipment and sensors:

- GSR and PPG sensor, Shimmer3 GSR+ Unit (Shimmer Sensing), a wireless sensor with Bluetooth that records GSR and PPG data locally on an SD card. The GSR is recorded via two single-use gel-type electrodes, while the PPG via an earlobe clip optical sensor;

- Eye tracker, Pupil Core (from Pupil Labs), which is an open-source eye-tracking platform connected via USBC. We use a single eye tracker on the right eye;

- Camera-based motion capture system from Motion Analysis, with 11 cameras;

- 4 Bertec force plates, synchronized with the motion capture system. 


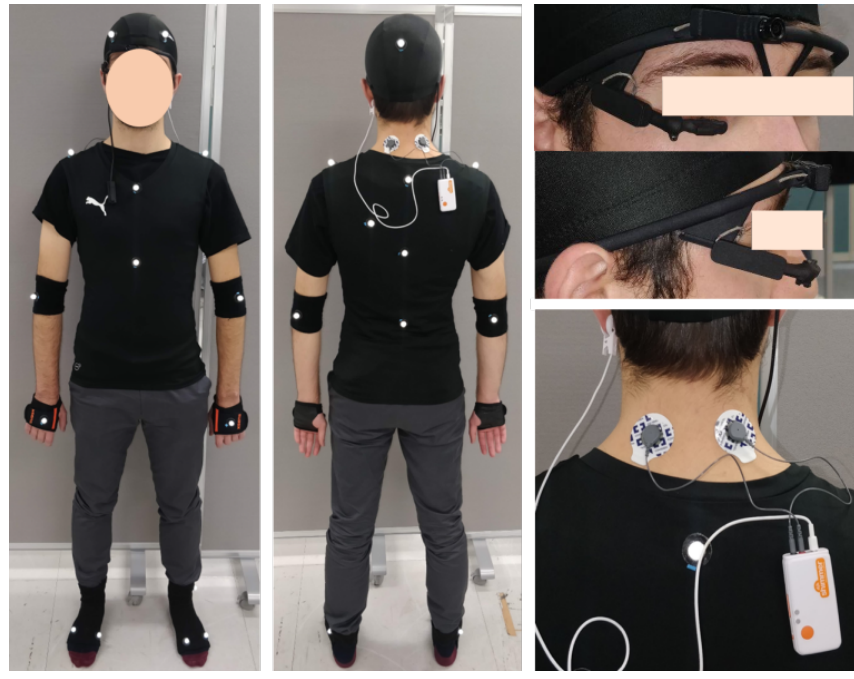

Fig. 3: Motion capture markers (23 in total), Pupil Core eye tracker, and Shimmer3 GSR+ Unit for GSR and PPG.

Typical locations for the electrodes of the GSR are the palm of the hands, however, given the interactive nature of our experiment, the electrodes have been positioned at the back of the neck (see Fig. 3), which also provides reliable measurements [29].

For the motion capture, we use a set of 23 markers: three on the head, one on each hand and two on each elbow, two on the front of the torso, four on the back and one on each shoulder, and three on each foot. All the markers are prepositioned on wearables: gloves, shirt, cap, and socks that can be worn on top of the users' clothes, such that the users can feel more comfortable without the need of changing clothes. The positioning of all the sensors and markers is shown in Fig. 3 All the data are synchronized via the Cortex software (Motion Analysis) and sampled at $100 \mathrm{~Hz}$, except for the eye tracker which is sampled at $125 \mathrm{~Hz}$ and the force plates at $500 \mathrm{~Hz}$.

\section{Experiment protocol}

Every experiment follows strictly the same protocol. Before the experiment, the participants read the experiment protocol which explains the setup and the procedure by themselves, to minimize the interaction with the instructor. The participants are then asked to watch a pre-recorded video with audio and subtitles in Japanese, explaining how to interact with the robot and how to play the game. The participants are not informed about the possibility of the robot to perform independent actions, as we are interested in the natural reaction to unexpected actions by the robot on the user. The protocol is provided online at [39].

Then the experiment proceeds as follows:

1) The participant completes the simplified version of the Big Five personality traits questionnaire consisting of 15 questions.

2) The participant is equipped with sensors and markers.

3) The participant rests for about 10 minutes during which the GSR and PPG baselines are recorded.

4) The participant performs two trial sessions, during which the robot is completely passive, i.e. no active force is applied. These sessions serve the user to get used to the robot.

5) The active session, involving the active force, is performed three times consecutively. The number of sessions has been chosen as a trade-off between making the users used to the experiment and keeping them motivated to play the game.

6) At the end of all sessions, the two questionnaires about robot perception are asked to be completed, CH-33 [4], and GSQ [35], one after the other, with the questions of each presented in a randomized order.

During each session, the record of the data starts about 10 seconds before the participants start the interaction with the robot, and ends about 10 seconds after the last interaction. This is to ensure that signals such as GSR and PPG are at their baseline levels before and after each session.

The total of 5 sessions lasts about 20 minutes, where a single session (trial or active) lasts about 2-3 minutes, during which the participants play the game that consists of 3 rounds, lasting 30 seconds each. The game can be restarted and the participants are asked to keep playing until instructed otherwise. Since the game is a task for the participants to interact with the robot, the winning and/or losing is not the goal of the experiment. In this paper, we set $\Delta T_{a}=1$ and $\Delta T=5$, so during each session, about 5-15 active forces may occur. Each experiment is video-recorded from two perspectives, from the front and the back.

All our experiments have been approved by the local ethics committee at the National Institute of Advanced Industrial Science and Technology (AIST) in Tsukuba, Japan. Before the experiment, participants have received proper information and given informed consent to participate in the study.

\section{Method AND RESUltS}

We performed the experiment with a total of 23 participants, of which only the data of 17 participants were usable due to technical issues, e.g. sensors not recording, unexpected external interferences. Of these 17 participants, 6 are females and 11 are males, with ages between 20 and 33 years old. All the participants are Japanese, born and raised in Japan. From a pre-experiment questionnaire (see additional material), all the participants have no or very little prior experience with robots in general.

\section{A. Data collection and post-process}

We collected data from the questionnaires presented in section II-A and all the sensors listed in section II-C. following the protocol detailed in section II-D

Motion capture data have been processed with Cortex software (Motion Analysis) first, then post-processed with DhaibaWorks [41]. In DhaibaWorks we use a human model that is scaled according to the height, weight, and gender of each participant, and the Sawyer model that is the same used in the robot controller. The human model motions are obtained via inverse kinematics by mapping recorded marker positions to virtual markers, while the robot motions by using the recorded joint angles. From DhaibaWorks we 


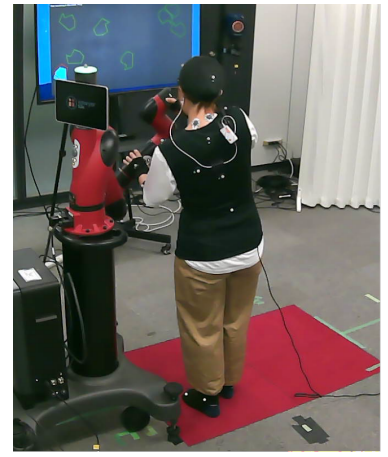

(a) Close

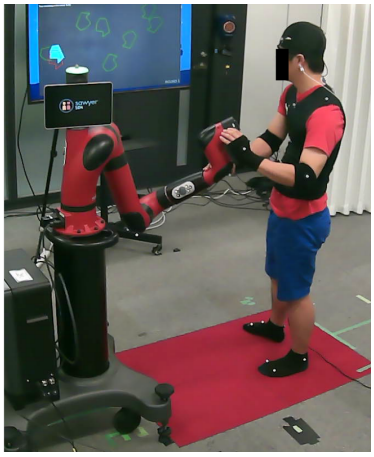

(b) Far
Fig. 4: Two participants with different behaviors.

extracted the poses of the participants, including specific body parts such as head, hands, and feet, from which we computed distances between the participant and the robot. We are interested in these data as participants showed overall different attitudes to the robot. Fig. 4 shows two examples. The participant in Fig. 4a keeps the EE very close to the face during part of the experiment, prefers to stand facing the screen, and uses one hand on the cuff and one hand to hold the arm of the robot. On the contrary, the participant in Fig. 4b keeps a far distance from the robot by putting both hands on the EE, and prefers to face the robot looking at the screen by turning the head. This can be seen better in the video attachment.

From the eye tracker, we can extract data such as gaze direction, EBR, and EBD. The gaze direction is used to determine where the participants are looking at, i.e. at the robot, at the screen, or elsewhere. Specifically, we use collision detection [42] to categorize the gaze location, by modeling the gaze as a cone directed as the gaze direction with an angle of $10^{\circ}$. We compute the total gazing time to each location only during three time intervals: the play of the game, before starting, and after ending the interaction, given that for the rest of the session, the user may be looking at the robot due to the necessity of using the buttons to restart/reset the game, and this is not relevant for our analysis.

From the Shimmer3 sensor, we obtained the GSR and PPG data, the latter being post-processed to obtain the PRV. Differently from the rest of the data, for GSR and PPG we have a pre-experiment record of the baseline that is used to evaluate the changes that occurred during the experiment.

\section{B. Factor extraction}

From the above-mentioned data, we extracted a series of factors, mainly based on statistical analysis, including percentage differences between stimuli (i.e. active force application) and the average of each session or the baseline (GSR, PRV). Given H1, we considered the active force application by considering a window of 3 seconds from the start of the stimuli, to which we will refer as action window. For the analysis, only the second trial session and the 3 active sessions (4 out of the 5 sessions) have been considered, the first trial session has not been considered due to the high amount of other external stimuli that affected the experiment, e.g. frequent help from the instructor.
A list of factors is shown in Table [ The factors in Group 1 are computed for all sessions (considering each session independently), while the factors of Group 2 are computed only for the active sessions, as they refer to percentage differences between the action window and the average during the session. The participants' gender, personality, and questionnaire outcomes are also included as part of the factors (not shown in Table IT). The post-experiment questionnaire $\mathrm{CH}-33$ consists of 33 questions rated on a 7 points Likert scale and projected onto 6 factors, as shown in Fig. 5a while GSQ is a series of 5 questionnaires rated on a 5 points semantic differential scale, resulting in 5 factors as shown in Fig. 5b. The personality test projects into 5 factors on a scale of $0-100$, as shown in Fig. 5c. For the details of these factors, please refer to [4], [35], and [38], respectively. From Fig. 5] we can observe that there is no specific trend of the questionnaire outcomes, indicating that the participants had overall different perceptions of the robot. This confirms that it might be interesting to analyze the correlation between the factors to identify relationships between perceptions, personality, and measurements, as per $\mathbf{H 2}$.

\section{Relevant correlations}

As most of the collected data are not Gaussian, we used Spearman's correlation for non-parametric data. In particular, the following correlations have been carried out to test our hypotheses:

- Personality and gender with the questionnaire outcomes;

- Personality, gender, and questionnaire outcomes with all the extracted factors for all the sessions;

- Personality, gender, and questionnaire outcomes with the percentage difference between the average of each factor over the active sessions and the trial session (ED factors).

We considered as relevant those that scored correlation coefficient $|r|>0.6$ and $\mathrm{p}$-value $p<0.05$, and that show interesting trends over the sessions (e.g. present in more than one session). These factors are reported in Table II. We ran the T-test for repeated samples on the factors of Group 1, for each active session with respect to the trial session, in order to verify possible significant changes when the active force is exerted. From the tests, most of the factors did not show significant differences, mostly with $p>0.5$. A few factors showed significant difference with only one session with $p<0.1$, while we verified that GSR diff showed $p<0.1$ for all three active sessions.

From Table II we can see that the correlation between Extraversion and CoP is consistent over all the sessions with negative correlation factors, indicating that extrovert participants approached the robot closer compared to the introvert ones. Anthropomorphism and Animacy from GSQ also show relevant correlation with GSR diff, with positive correlation indicating that the more the participants perceived the robot as anthropomorphic and animated, the lower their GSR level was during the sessions with respect to the baseline. However, the correlation weakens as the participants go through the sessions, with Anthropomorphism scoring 


\begin{tabular}{|c|l|}
\hline \multicolumn{1}{|c|}{ Factor } & Explanation \\
\hline Group 1: average over each session including the trial session \\
\hline CoP & $\begin{array}{l}\text { Center of pressure distance between the participant and } \\
\text { the robot base }\end{array}$ \\
\hline Walking & Amount of walking as total number of steps/time \\
\hline Head dist & Distance between head and robot EE \\
\hline Head ort & Orientation of the head \\
\hline Feet dist & Distance between the feet and robot base \\
\hline Feet ort & Orientation of the feet \\
\hline Hand ext & $\begin{array}{l}\text { Extension of the arms as sum of distances from each } \\
\text { hand to the torso, normalized by height }\end{array}$ \\
\hline LHand loc & Location of the left hand, i.e. robot arm, wrist, or EE \\
\hline Cuff frq & Frequency of pressing the interaction cuff \\
\hline Gaze time diff game & $\begin{array}{l}\text { Difference between gazing time at the robot and the } \\
\text { screen during the game }\end{array}$ \\
\hline Gaze time diff before & $\begin{array}{l}\text { Difference between gazing time at the robot and the } \\
\text { screen before starting the interaction }\end{array}$ \\
\hline Gaze time diff after & $\begin{array}{l}\text { Difference between gazing time at the robot and the } \\
\text { screen after ending the interaction }\end{array}$ \\
\hline GSR diff & Difference of GSR between the baseline and the session \\
\hline PRV diff & Difference of PRV between the baseline and the session \\
\hline $\begin{array}{l}\text { Group 2: percentage difference between action window and the rest of the } \\
\text { session, for each active session }\end{array}$ \\
\hline EBR diff AF & Eye blinking rate \\
\hline EBD diff AF & Eye blinking duration \\
\hline Pupil diff AF & Pupil dimension \\
\hline EE force diff AF & Forces measured at the robot EE \\
\hline GRF force diff AF & Ground reaction forces, normalized with body weight \\
\hline
\end{tabular}

TABLE I: All factors excluding personality, gender, and questionnaires. Factors in Group 1 are computed for all the sessions, while those in Group 2 excluding the trial session. In the table, "diff" means percentage difference, while "AF" refers to the average during the action windows.

\begin{tabular}{|c|c|c|}
\hline Factor 1 & Factor 2 & Correlation coeff \\
\hline \multicolumn{3}{|l|}{ Trial session } \\
\hline Extraversion & $\mathrm{CoP}$ & $-0.74 * *$ \\
\hline Anthropomorphism & GSR diff & $0.71 *$ \\
\hline Animacy & GSR diff & $0.69 *$ \\
\hline \multicolumn{3}{|l|}{ Active Session 1} \\
\hline Extraversion & $\mathrm{CoP}$ & $-0.73 *$ \\
\hline Anthropomorphism & GSR diff & $0.66 *$ \\
\hline Animacy & GSR diff & $0.72 * *$ \\
\hline \multicolumn{3}{|l|}{ Active Session 2} \\
\hline Extraversion & $\mathrm{CoP}$ & $-0.73 * *$ \\
\hline Animacy & GSR diff & $0.65 *$ \\
\hline \multicolumn{3}{|l|}{ Active Session 3} \\
\hline Extraversion & $\mathrm{CoP}$ & $-0.81 * *$ \\
\hline \multicolumn{3}{|c|}{ Difference between average of active and trial session } \\
\hline Extraversion & Hand Ext ED & 0.61 \\
\hline Performance & Walking ED & $0.71 *$ \\
\hline Acceptance & Walking ED & $0.67 *$ \\
\hline Toughness & Walking ED & $0.65 *$ \\
\hline Toughness & Feet Ort ED & $0.65 *$ \\
\hline Likeability & Walking ED & 0.64 \\
\hline Animacy & PRV Diff ED & $-0.67 *$ \\
\hline \multicolumn{3}{|c|}{ Personality and questionnaires } \\
\hline Openness & Toughness & -0.64 \\
\hline Openness & Agency & $0.77 * *$ \\
\hline Openness & Likeability & $-0.68 *$ \\
\hline Agreeableness & Agency & $0.67 *$ \\
\hline
\end{tabular}

TABLE II: Extracted relevant factors with correlation coefficient $>0.6$ and $\mathrm{p}$-value $<0.05$. Those with $*$ indicate $\mathrm{p}$-value $<$ 0.005 , and $* *$ indicates $\mathrm{p}$-value $<0.001$. "ED" refers to the percentage difference between the average of the three active sessions and the trial session.

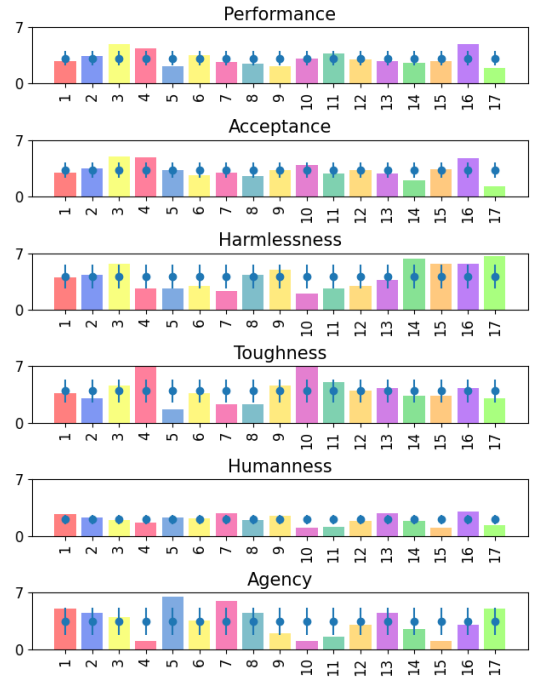

(a) $\mathrm{CH}-33$

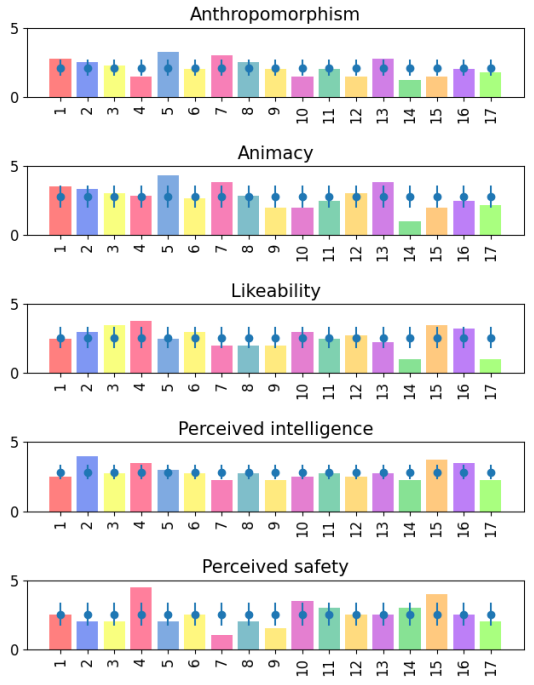

(b) GSQ

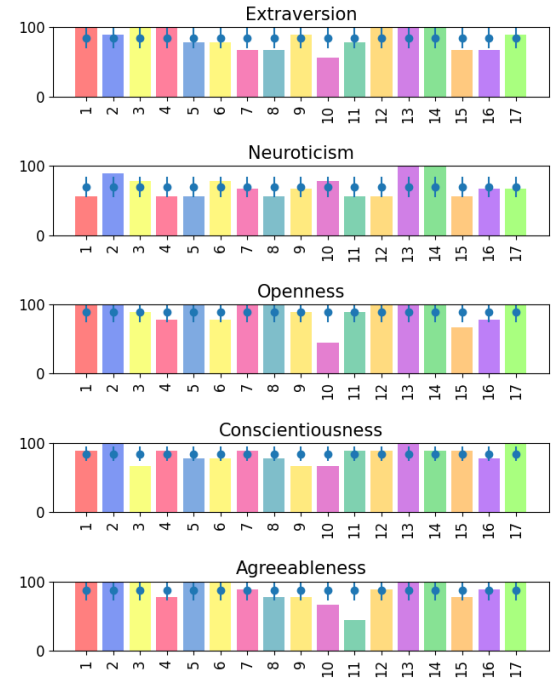

(c) Personality

Fig. 5: Results of the questionnaires CH-33 (a) on a scale of 1-7, GSQ (b) on a scale of 1-5, and personality (c) on a scale of 0-100, for the 17 participants considered in this study, the error bar shows the mean and standard deviations of the factor for all the participants.

$|r|<0.6$ for active sessions 2 and 3 , and Animacy for active session 3. This trend indicates that the participants had variations in the GSR level over the sessions that were not strongly related to their perception of the robot.

In Table II we can also observe a few correlations with the ED factors. In particular, more extrovert participants tend to have a wider extension of their arms during the active sessions with respect to the trial session. This may indicate that extroverts tend to impose on the robot more with their body with respect to introverts. Participants who perceived the robot as tougher (less likely to break down), more performant, acceptable, and likable, walked more during the active sessions. Also, those who perceived the robot as tougher, had feet more oriented towards the screen during the active sessions. High scores in these factors indicate overall a higher confidence on the robot capabilities as well as comfort they felt during the interaction with the robot, therefore, these correlations may indicate that the 
participants who felt overall more comfortable and confident in the robot, felt more comfortable to walk around while manipulating the robot (e.g. the second participant shown in the video attachment) and/or also preferred to be more oriented towards the screen rather than the robot (e.g. the first participant shown in the video attachment), which may also indicate higher trust in the robot.

We also obtained relevant correlations between participants' personality and the way they perceive the robot. Specifically, the more open the participants, the less they perceived the robot as tough and likable, and the more they perceived the robot as an agent. The more the participants are agreeable, the more they perceived the robot as an agent.

\section{DISCUSSION}

H1 states that the active force can cause measurable alterations in the participants' data. However, from the T-test, only the GSR diff showed significant changes, implying that possible alterations are not measurable via the other factors presented in Table I. This also may mean that the ED factors should be interpreted with caution. $\mathbf{H 2}$ states that the measurable data can be related to the questionnaire outcomes. However, only two of the factors in Table I showed relevant correlations, namely the CoP and GSR diff. The former indicates that extroverted people seem to be approaching the robot from a closer distance, and the latter that the people who perceive the robot as anthropomorphic and animated could be more stressed/anxious when interacting with the robot with respect to resting conditions.

Even if we could not find an adequate number of factors satisfying our hypotheses, we cannot neglect the relevance of the obtained results. The outcomes give important insights into active pHRI experiments. We adopted a game-style task and a simple disturbance-style action in order to have a smaller number of variables for which the isolation of factors could have been less affected by random noise. However, this might have been the main limitation of our experiment, as the action seems to have not been strongly perceived by the participants. Therefore, in the design of active pHRI experiments, it could be advisable to use different types of "actions", for example, a collaborative one or one that has different objectives. We also tried to break down the different participants' attitudes observed in section III-A. however, these did not show up significantly in the resulting relevant factors, therefore, a different way of quantifying such attitudes may be advisable. GSQ was chosen due to its popularity in HRI, however, Animacy and Anthropomorphism showed high correlation, and as a matter of fact, it has been shown that there are some flaws in the GSQ [43]. We think this may affect the overall outcome of the questionnaire, so it may not be well suited for active pHRI. Personality appears to give interesting insights into the way participants approach and behave with the robot. As a matter of fact, the relationship between personality and personal spatial zones have been studied in literature [27], and the correlations between Extraversion and CoP and hand extension, both go in the direction of a possible relationship between the two. Therefore, we consider the investigation of personality and distances to be important.

It is also worth noting that none of the factors showed relevant correlations with gender, indicating that in active pHRI experiments, gender may not be influential. The restriction on cultural background has been introduced to avoid cultural dependencies, however, it also represents a limitation for which the outcomes may not be generalizable for different cultural backgrounds. Last but not least, the participants have been recruited via open calls, many of them seemed to have little interest in the content of the experiment itself, and many had several experiences with other types of laboratory experiments as well. We think that this has effects on the outcomes that are not negligible, therefore, we deem it important to consider also the participants' habituation levels and mindsets towards laboratory experiments.

\section{CONCLUSIONS AND FUtURE DEVELOPMENTS}

In this study, we were interested in analyzing the perceptions and state of humans with respect to an active physical interaction with a robot. We hypothesized that this action can cause alterations in measurable physical and physiological data, and that these data can be related to personality, gender, as well as the perception of the users. However, results did not show many correlations with the extracted factors.

This study was an exploratory study meant as a first attempt to verify the possibility of identifying measurable interaction factors that could be useful for the development of active pHRI controllers, by combining tools from both pHRI and sHRI. Despite only a few factors having been uncovered, we obtained insightful observations and advice for the design of active pHRI, based on the advantages and pitfalls of our experiment. We consider these findings useful for researchers who may be interested in developing active pHRI experiments, while with future more consistent findings on interaction factors, we believe it will be fundamental for those who develop controllers for pHRI applications, as measurable quantities are necessary as inputs for controllers. Assuming that the interactions have specific objectives (e.g. helping to stand up or to lift pieces), the interaction factors could be used as weighted objectives to modify the motions of the robot according to the users' state. However, the inclusion of these factors in a control framework is out of the scope of this paper and will be considered for future work.

The findings of this paper will be taken into account in our next experiment. We will also target a higher number of participants to collect more consistent data, including qualitative data that were not considered in this study, which could help understanding better the results. To be able to identify as many factors as possible, we deemed necessary, for the time being, to use many sensors and equipment. However, to target real-world scenarios, less invasive setups will also be considered. From the future experiments, we expect to be able to extract further factors that can relate the human physiological, physical, and mental state with respect to different robot actions. Furthermore, Affective Computing methodologies, classification and/or factor analysis on the 
data may lead to interesting outcomes and will be considered for further studies.

\section{REFERENCES}

[1] ISO, 15066-Robots and robotic devices-Collaborative robots, ISO (International Organization for Standardization) TS (Technical Specification)

[2] S. Haddadin, S. Haddadin, A. Khoury, T. Rokahr, S. Parusel, R. Burgkart, A. Bicchi, A. Albu-Schäffer, On making robots understand safety: Embedding injury knowledge into control, The International Journal of Robotics Research 31 (13) (2012) 1578-1602.

[3] A. Bicchi, M. A. Peshkin, J. E. Colgate, Safety for physical humanrobot interaction, Springer Handbook of Robotics (2008) 1335-1348.

[4] H. Kamide, Y. Mae, K. Kawabe, S. Shigemi, M. Hirose, T. Arai, New measurement of psychological safety for humanoid, in: ACM/IEEE International Conference on Human-Robot Interaction, 2012, pp. 49 56.

[5] M. Khoramshahi, A. Laurens, T. Triquet, A. Billard, From human physical interaction to online motion adaptation using parameterized dynamical systems, in: IEEE/RSJ International Conference on Intelligent Robots and Systems, 2018, pp. 1361-1366.

[6] E. Lamon, L. Peternel, A. Ajoudani, Towards a prolonged productivity in industry 4.0: A framework for fatigue minimisation in robotrobot co-manipulation, in: IEEE/RAS International Conference on Humanoid Robots, 2018, pp. 1-6.

[7] D. J. Agravante, A. Cherubini, A. Bussy, P. Gergondet, A. Kheddar, Collaborative human-humanoid carrying using vision and haptic sensing, in: IEEE International Conference on Robotics and Automation, 2014, pp. 607-612. doi:10.1109/ICRA.2014.6906917

[8] M. Gienger, D. Ruiken, T. Bates, M. Regaieg, M. MeiBner, J. Kober, P. Seiwald, A.-C. Hildebrandt, Human-robot cooperative object manipulation with contact changes, in: IEEE/RSJ International Conference on Intelligent Robots and Systems, 2018, pp. 1354-1360.

[9] M. S. Erden, T. Tomiyama, Human-intent detection and physically interactive control of a robot without force sensors, IEEE Transactions on Robotics 26 (2) (2010) 370-382.

[10] Y. Li, S. S. Ge, Human-robot collaboration based on motion intention estimation, IEEE/ASME Transactions on Mechatronics 19 (3) (2013) 1007-1014.

[11] Y. Tirupachuri, G. Nava, C. Latella, D. Ferigo, L. Rapetti, L. Tagliapietra, F. Nori, D. Pucci, Towards partner-aware humanoid robot control under physical interactions, in: Proceedings of SAI Intelligent Systems Conference, Springer, 2019, pp. 1073-1092.

[12] M. Babaiasl, S. H. Mahdioun, P. Jaryani, M. Yazdani, A review of technological and clinical aspects of robot-aided rehabilitation of upper-extremity after stroke, Disability and Rehabilitation: Assistive Technology 11 (4) (2016) 263-280.

[13] K. W. Lee, S. B. Kim, J. H. Lee, S. J. Lee, J. W. Kim, Effect of robotassisted game training on upper extremity function in stroke patients, Annals of Rehabilitation Medicine 41 (4) (2017) 539-546.

[14] A. Weiss, R. Bernhaupt, M. Tscheligi, E. Yoshida, Addressing user experience and societal impact in a user study with a humanoid robot, in: Proceedings of the Symposium on New Frontiers in Human-Robot Interaction, Citeseer, 2009, pp. 150-157.

[15] S. Ivaldi, S. M. Anzalone, W. Rousseau, O. Sigaud, M. Chetouani, Robot initiative in a team learning task increases the rhythm of interaction but not the perceived engagement, Frontiers in Neurorobotics 8 (2014) 5.

[16] H. Kamide, K. Kawabe, S. Shigemi, T. Arai, Anshin as a concept of subjective well-being between humans and robots in japan, Advanced Robotics 29 (24) (2015) 1624-1636.

[17] J. Schmidtler, K. Bengler, F. Dimeas, A. Campeau-Lecours, A questionnaire for the evaluation of physical assistive devices (quead) Testing usability and acceptance in physical human-robot interaction, in: IEEE International Conference on Systems, Man, and Cybernetics, 2017, pp. 876-881.

[18] T. Arai, R. Kato, M. Fujita, Assessment of operator stress induced by robot collaboration in assembly, CIRP (International Academy for Production Engineering) annals 59 (1) (2010) 5-8.

[19] P. Rani, N. Sarkar, C. A. Smith, L. D. Kirby, Anxiety detecting robotic system-towards implicit human-robot collaboration, Robotica 22 (1) (2004) 85-95.

[20] A. Cruz-Maya, R. Agrigoroaie, A. Tapus, Improving user's performance by motivation: Matching robot interaction strategy with user's regulatory state, in: International Conference on Social Robotics, Springer, 2017, pp. 464-473.
[21] S. M. Anzalone, S. Boucenna, S. Ivaldi, M. Chetouani, Evaluating the engagement with social robots, International Journal of Social Robotics 7 (4) (2015) 465-478.

[22] J. Baraglia, M. Cakmak, Y. Nagai, R. P. Rao, M. Asada, Efficient human-robot collaboration: when should a robot take initiative?, The International Journal of Robotics Research 36 (5-7) (2017) 563-579.

[23] J. Tao, T. Tan, Affective computing: A review, in: International Conference on Affective Computing and Intelligent Interaction, Springer, 2005, pp. 981-995.

[24] T. L. Chen, C.-H. A. King, A. L. Thomaz, C. C. Kemp, An investigation of responses to robot-initiated touch in a nursing context, International Journal of Social Robotics 6 (1) (2014) 141-161.

[25] A. E. Block, K. J. Kuchenbecker, Softness, warmth, and responsiveness improve robot hugs, International Journal of Social Robotics 11 (1) (2019) 49-64.

[26] N. T. Fitter, K. J. Kuchenbecker, Synchronicity trumps mischief in rhythmic human-robot social-physical interaction, in: Robotics Research, Springer, 2020, pp. 269-284.

[27] M. L. Walters, K. Dautenhahn, R. Te Boekhorst, K. L. Koay, C. Kaouri, S. Woods, C. Nehaniv, D. Lee, I. Werry, The influence of subjects' personality traits on personal spatial zones in a human-robot interaction experiment, in: IEEE International Workshop on Robot and Human Interactive Communication., 2005, pp. 347-352.

[28] M. Lajante, O. Droulers, T. Dondaine, D. Amarantini, Opening the "black box" of electrodermal activity in consumer neuroscience research., Journal of Neuroscience, Psychology, and Economics 5 (4) (2012) 238

[29] M. van Dooren, J. H. Janssen, et al., Emotional sweating across the body: Comparing 16 different skin conductance measurement locations, Physiology \& Behavior 106 (2) (2012) 298-304.

[30] J. Moraes, M. Rocha, G. Vasconcelos, J. Vasconcelos Filho, V. de Albuquerque, A. Alexandria, Advances in photopletysmography signal analysis for biomedical applications, Sensors 18 (6) (2018) 1894.

[31] F. Mokhayeri, M. Akbarzadeh-T, Mental stress detection based on soft computing techniques, in: IEEE International Conference on Bioinformatics and Biomedicine, 2011, pp. 430-433.

[32] R. Zangróniz, A. Martínez-Rodrigo, M. López, J. Pastor, A. Fernández-Caballero, Estimation of mental distress from photoplethysmography, Applied Sciences 8 (1) (2018) 69.

[33] S. Benedetto, M. Pedrotti, L. Minin, T. Baccino, A. Re, R. Montanari, Driver workload and eye blink duration, Transportation research part F: traffic psychology and behaviour 14 (3) (2011) 199-208.

[34] J. Oh, S. Y. Jeong, J. Jeong, The timing and temporal patterns of eye blinking are dynamically modulated by attention, Human Movement Science 31 (6) (2012) 1353-1365.

[35] C. Bartneck, D. Kulić, E. Croft, S. Zoghbi, Measurement instruments for the Anthropomorphism, Animacy, Likeability, Perceived Intelligence, and Perceived Safety of Robots, International Journal of Social Robotics 1 (1) (2009) 71-81.

[36] A. Weiss, C. Bartneck, Meta analysis of the usage of the Godspeed Questionnaire Series, in: IEEE International Symposium on Robot and Human Interactive Communication, 2015, pp. 381-388.

[37] U. Morsunbul, Human-robot interaction: How do personality traits affect attitudes towards robot?, Journal of Human Sciences 16 (2) (2019) 499-504.

[38] G. M. Hurtz, J. J. Donovan, Personality and job performance: The Big Five revisited., Journal of Applied Psychology 85 (6) (2000) 869

[39] Experiment protocol and questionnaires, https://github.com/ hu-yue/HU RAL2020 questionnaires

[40] J. Vaillant, A. Kheddar, H. Audren, F. Keith, S. Brossette, A. Escande, K. Bouyarmane, K. Kaneko, M. Morisawa, P. Gergondet, et al., Multi-contact vertical ladder climbing with an HRP-2 humanoid, Autonomous Robots 40 (3) (2016) 561-580.

[41] Y. Endo, M. Tada, M. Mochimaru, Dhaiba: development of virtual ergonomic assessment system with human models, in: Proceedings of The 3rd International Digital Human Symposium, 2014.

[42] M. Benallegue, A. Escande, S. Miossec, A. Kheddar, Fast c1 proximity queries using support mapping of sphere-torus-patches bounding volumes, in: IEEE International Conference on Robotics and Automation, 2009, pp. 483-488. doi:10.1109/ROBOT.2009.5152722

[43] C. M. Carpinella, A. B. Wyman, M. A. Perez, S. J. Stroessner, The robotic social attributes scale (rosas) development and validation, in: ACM/IEEE International Conference on human-robot interaction, 2017, pp. 254-262. 